\title{
INVESTIGATION OF THE ROUGHNESS OF THE AUSTRALIAN GRAVITY FIELD USING STATISTICAL, GRAPHICAL, FRACTAL AND FOURIER POWER SPECTRUM TECHNIQUES
}

\author{
Kefei Zhang $^{1}$ and W.E. Featherstone ${ }^{2}$ \\ ${ }^{1}$ Department of Geospatial Science, RMIT University, GPO Box 2476V, Melbourne \\ 3001, Australia; \\ ${ }^{2}$ Western Australian Centre for Geodesy, Curtin University of Technology, GPO Box \\ U1987, Perth, WA 6845, Australia.
}

\begin{abstract}
As one of the world's oldest continents, Australia has experienced a complicated geological and geomorphological history. As such, the gravity field of Australia behaves differently to that reported in other countries. This paper investigates the relative roughness of three types of gravity anomaly (free-air, refined Bouguer and topographic-isostatic) using statistical and graphical comparisons, power spectrum analysis, and the Hurst fractal technique. Gravity and height profiles in the Hamersley Ranges, central Australia and the Snowy Mountains, which exhibit some extreme features, have been selected to compare these methods. The statistical comparisons are the most informative tools for measuring the relative roughness of the gravity field. Also, not one of the free-air, refined Bouguer or topographic-isostatic gravity anomaly is consistently the smoothest type in Australia, which is due to a combination of a lack of accurate topographic density information and a relatively long-wavelength topography so that the terrain correction cannot always smooth the computed gravity anomalies. It is recommended that the smoothest type of gravity anomaly in each area be used to grid gravity data prior to geoid computation.
\end{abstract}

\section{INTRODUCTION}

With the advent of the Global Positioning System (GPS), there has been an increased demand for precise geoid models with which to transform GPS-derived ellipsoidal heights to orthometric heights. This has triggered a large amount of geoid-related research over the past decade. Generally, large amounts of data are involved in geoid determination, particularly terrain correction computations. For such computations over large areas, it is often convenient if the quantity to be analysed is decomposed as a sum of components, a process known as spectral analysis. The Fast Fourier Transformation (FFT) and Fast Hartley Transform (FHT) techniques [4-6] have proven to be useful tools for the efficient evaluation of gravity field convolution integrals [14].

However, the FFT and FHT approaches normally require that gridded gravity data be utilised (eg $[\mathbf{1 1}, \mathbf{1 4}, \mathbf{1 6}])$. Therefore, the irregularly spaced gravity observations must be resampled onto a regular grid over the area of interest. The conventional approach to such gravity gridding (eg. [9]) is to smooth the gravity field using reductions (the remove stage), interpolate these smoothed quantities onto the desired grid (the compute stage), and then add back the appropriate gravity reduction (the restore stage). In almost all instances, refined Bouguer gravity anomalies are used for data smoothing purposes prior to gridding. However, if the reduced gravity data are not smooth after the remove stage, interpolation errors will result in the gravity grid, which then propagate into the computed gravimetric geoid. It is also important to acknowledge that, as well as the smoothness of the gravity anomaly, the effectiveness of the gridding algorithm is important, but will not be discussed in this paper.

This paper summarises an investigation by Zhang [17] into the features of the Australian gravity field in terms of relative roughness of various gravity anomaly types to determine which is the most appropriate for gridding gravity data prior to geoid computation. The 
methods used comprise fractal geometry and Fourier spectrum analysis, in addition to simple descriptive statistics and graphical visualisation. It will be shown that the gravity field in Australia is relatively complicated and does not adhere to the traditional axioms. Not one of the free-air, refined Bouguer or (Airy-Heiskanen) topographic-isostatic gravity anomalies is consistently the smoothest in Australia. This suggests that in order to grid the gravity field prior to geoid determination, some hybrid approach should be used, where the smoothest gravity anomaly type is used in different areas.

\section{DATA AND INVESTIGATIVE PROCEDURES}

Gravity data over the Australian continent were supplied in 1992 by the then Australian Geological Survey Organisation (AGSO; now called Geoscience Australia). This data set includes 526,091 land and 111,396 marine gravity observations. These data have been reformatted and validated using the procedures described in [8,17]. A digital file of approximately six million spot heights was supplied by the Australian Surveying and Land Information Group (AUSLIG; now the National Mapping Division of Geoscience Australia). These spot heights were combined with the gravity observation elevations, then gridded at a 1 $\mathrm{km}$ by $1 \mathrm{~km}$ resolution using continuos curvature splines in tension [15] to produce a digital elevation model (DEM). The various gravity anomaly types used in this study were calculated using the 1992 AGSO gravity database and this $1 \mathrm{~km}$ by $1 \mathrm{~km}$ DEM.

The Australian free-air gravity anomalies show a preponderance of negative features in the southwest and more positive features in the north and east, whereas a largely negative Bouguer gravity anomaly field is a prominent feature of the Australian gravity data. Due to these variable features of the Australian gravity field, three profiles were selected that exhibit different topographic and gravity anomaly features. Profile $\mathrm{P}_{1}$ is located in the Hamersley Ranges of Western Australia, profile $\mathrm{P}_{2}$ in central Australia, and profile $\mathrm{P}_{3}$ in the Snowy Mountains of eastern Australia (see Table 1 and Figure 1).

Table 1. Locations of the three test profiles in the Hamersley Ranges of Western Australia $\left(P_{1}\right)$, central Australia $\left(P_{2}\right)$, and the Snowy Mountains of eastern Australia $\left(P_{3}\right)$

\begin{tabular}{c|c|c|c}
\hline Profile & $\mathrm{P}_{1}$ & $\mathrm{P}_{2}$ & $\mathrm{P}_{3}$ \\
\hline Latitude & $25^{\circ} \mathrm{S}$ & $26^{\circ} \mathrm{S}$ & $36^{\circ} \mathrm{S}$ \\
\hline Longitude & $116^{\circ} \mathrm{E} \sim 120^{\circ} \mathrm{E}$ & $126^{\circ} \mathrm{E} \sim 132^{\circ} \mathrm{E}$ & $145^{\circ} \mathrm{E} \sim 149^{\circ} \mathrm{E}$ \\
\hline
\end{tabular}

The techniques used to determine which type of gravity anomaly is the smoothest over each profile are:

1. Simple statistics (i.e. maximum, minimum, mean and standard deviation);

2. Direct visual comparisons of graphs;

3. Power spectral analysis; and

4. Hurst fractal dimension computations. 


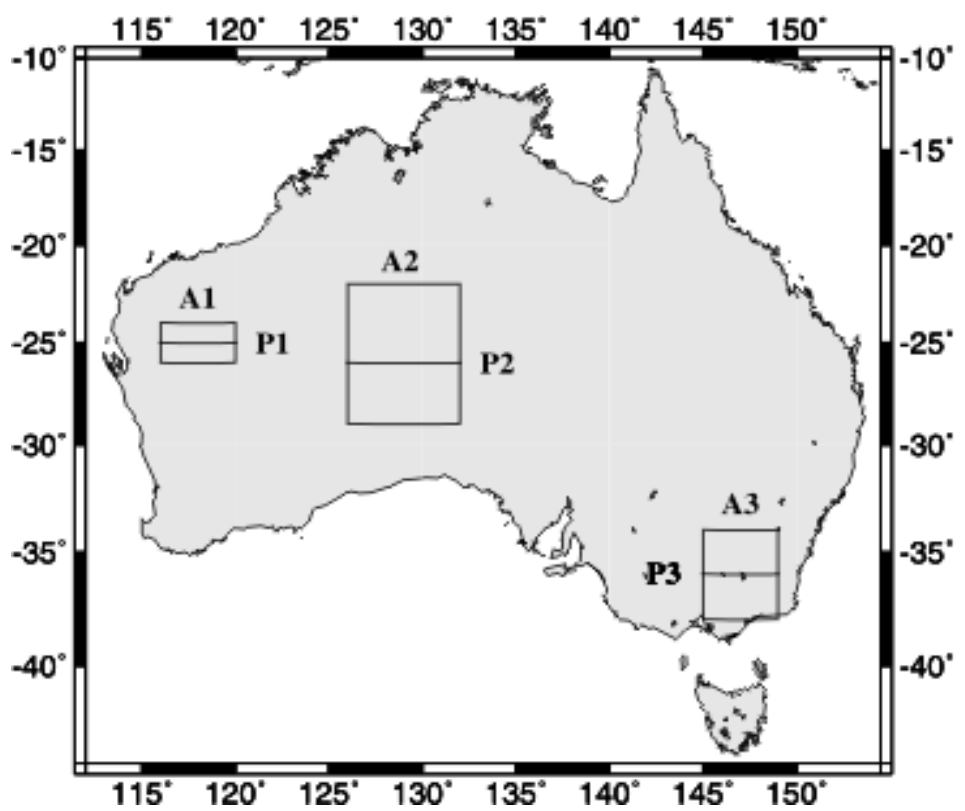

Fig. 1. Geographical location of the test profiles in the three typical areas of Australia (e.g. A1: the Hamersley Ranges, A2: central Australia and A3: the Snowy Mountains) (Linear projection)

The power spectral and fractal analysis techniques are well documented in the literature (eg. $[3,4,7])$. However, fractal analysis is a relatively new technique in geodesy, and thus offers a new paradigm for understanding the detailed structure and features of the Earth's gravity field. The fractal characterisation technique was initially applied to time-based phenomena (eg. $[13,12,3,2])$, but its better-known applications are to surfaces and profiles [3]. The underlying characteristic of a fractal set is the self-similarity of the scales in the sense that there are large and small scales that maintain some relation between them [7]. Of particular relevance to this study, the fractal dimension of a profile can be used to quantify its relative roughness, where the larger the fractal dimension, the rougher the profile and vice versa. Therefore, the fractal dimension offers an additional tool for measuring the relative roughness of various types of gravity anomaly.

There are many different approaches to estimate fractal dimensions (eg. [13,2]). The Hurst, or rescaled range analysis of fractals, method was chosen for this investigation because of its relative simplicity [10]. The basic idea behind the construction of the Hurst fractal is to use a log-log plot of the maximum differences of the quantities under investigation in a given window, versus the size of that window. Least-squares linear regression is then used to determine the slope of this log-log plot, from which the Hurst fractal dimension is subsequently determined. A useful description of the calculation and application of the Hurst fractal is given in [13].

The Hurst method is applied to gravity anomalies as follows: assuming that the gravity anomalies $(\Delta g)$ in a limited area follow self-similarity, the log maximum differences of the gravity anomalies $(\log \Delta g)$ and $\log$ of the differences of the distances between these $(\log L)$ for a given window size are related by

$$
S_{i}=\frac{\log _{10}\left(\Delta g_{i}\right)}{\log _{10}\left(L_{i}\right)}
$$

and for a profile, the Hurst fractal dimension is computed from

$$
D=2-\hat{S}
$$


where $S_{i}$ is the slope of the log-log graph corresponding to the $i^{\text {th }}$ window, $\hat{S}$ is the regression slope of the log-log plot with a root-mean-square regression error $\sigma, D$ is the Hurst fractal dimension, and $\Delta g_{i}$ is the maximum difference of the gravity anomalies within the $i^{\text {th }}$ window size $\left(L_{i}\right)$.

\section{TEST RESULTS AND ANALYSIS}

\section{Statistical Comparisons}

A preliminary indication of the relative roughness of the gravity anomalies over Australia can be seen by comparing the descriptive-statistical properties of two gravity anomaly types across the whole continent (Table 2). Topographic isostatic anomalies were not available over the whole continent.

Table 2. The statistics of gravity anomalies on land over Australia (units in mGal)

\begin{tabular}{c|c|c|c|c|c}
\hline & $\max$ & $\min$ & mean & rms & std \\
\hline Free-air & 172.89 & -121.10 & 0.40 & 25.31 & 25.10 \\
\hline Bouguer & 82.56 & -164.00 & -24.79 & 41.27 & 33.00 \\
\hline
\end{tabular}

Generally, the refined Bouguer gravity anomalies are expected to be smoother than the freeair gravity anomalies, because the Bouguer correction is assumed to remove the correlation of the free-air anomalies with topographic height (notwithstanding isostatic compensation). However, in Table 2 the standard deviation (std) of the Bouguer anomalies is higher than that of free-air anomalies over the Australian continent. This implies that the application of a constant topographical density cannot effectively remove the effect of the topography in Australia, and it is thus concluded that a complicated geological structure exists. However, isostatic compensation may also contribute to the above observation.

The relatively large standard deviation in Table 2 indicates that the Bouguer gravity anomalies are more variable on a continental scale, so could be expected to introduce larger interpolation errors than free-air anomalies if used during the gridding process. However, given that interpolation relies more upon the detailed structure of the quantity under investigation, the standard deviation of a continent-wide dataset does not necessarily indicate its detailed variability. Therefore, the result in Table 2 cannot be used solely to infer the detailed roughness of the gravity anomaly types prior to gridding. Instead, a relative measure of the detailed roughness of the gravity anomaly types is arguably more useful to determine which is most suited to gridding.

Table 3. The statistics of $(F A=$ free-air, $B G=$ refined Bouguer, $T I=$ topographicisostatic) gravity anomalies over the three test profiles

\begin{tabular}{l|c|c|c|c|c|c|c|c|c}
\hline \multirow{2}{*}{$\begin{array}{l}\text { Statistics } \\
\text { (mGal) }\end{array}$} & \multicolumn{3}{|c|}{ Profile $\mathrm{P}_{1}$} & \multicolumn{3}{c|}{ Profile $\mathrm{P}_{2}$} & \multicolumn{3}{c}{ Profile $\mathrm{P}_{3}$} \\
\cline { 2 - 10 } & FA & BG & TI & FA & BG & TI & FA & BG & TI \\
\hline max-min & 61.3 & 74.6 & 51.2 & 128.0 & 219.5 & 137.4 & 161.0 & 71.7 & 53.6 \\
\hline mean & 8.8 & -40.2 & 4.8 & -31.4 & -48.4 & -37.0 & 2.7 & -20.7 & 1.8 \\
\hline rms & 27.3 & 64.6 & 25.7 & 99.0 & 48.2 & 52.1 & 24.8 & 25.6 & 12.7 \\
\hline std & 15.8 & 9.8 & 12.9 & 15.4 & 71.5 & 15.2 & 23.5 & 18.6 & 10.2 \\
\hline
\end{tabular}

Following the above discussion, this study now concentrates on the three profiles in Table 1 and Figure 1. Topographic-isostatic gravity anomalies were also computed for 
these profiles and compared statistically to determine whether these appear less variable than the Bouguer and free-air anomalies (Table 3).

Comparing the standard deviations for each of the three profiles in Table 3 shows that there is no indication that any gravity anomaly type is consistently the smoothest. For example, in profile $\mathrm{P}_{1}$, the Bouguer anomalies are the least variable, whereas in profile $\mathrm{P}_{3}$, the topographic-isostatic gravity anomalies are the least variable (Table 3). However, simple statistics do not provide conclusive evidence in their own right as to the very detailed roughness of these gravity anomaly types, because the computed standard deviation is also a function of the length of the profile.

For example, the conclusions reached from the free-air and Bouguer gravity anomalies in Table 2 are at odds with that reached from Table 3. Given that gridding is more sensitive to the detailed properties of the gravity anomalies, the area over which the simple statistics are computed deserves further attention. Therefore, the alternative approaches of graphical, spectral and fractal analyses are used to support or refute the above inferences made about the relative roughness of the gravity anomaly types.

\section{Graphical Comparisons}

The free-air, refined Bouguer and topographic-isostatic gravity anomalies and terrain height are plotted for profiles $P_{1}, P_{2}$ and $P_{3}$ in Figures 2 to 4 , respectively. Through a visual inspection of the profiles in Figure 3, for example, the Bouguer anomalies are not necessarily smoother than the free-air anomalies (eg. the region $126.0^{\circ} \mathrm{E} \sim 128.0^{\circ} \mathrm{E}$ ). This is most probably due to a combination of the use of a constant topographic density $\left(2670 \mathrm{kgm}^{-3}\right)$ to compute the refined Bouguer gravity anomaly, and that the topography is of longer wavelength than the gravity anomalies (Figure 3) and thus cannot provide any detailed smoothing of the gravity field. This alone suggests that, as long as a density model is not used, Bouguer gravity anomalies are not necessarily the optimum type of gravity anomaly to use for interpolation in this, and possibly other, regions.

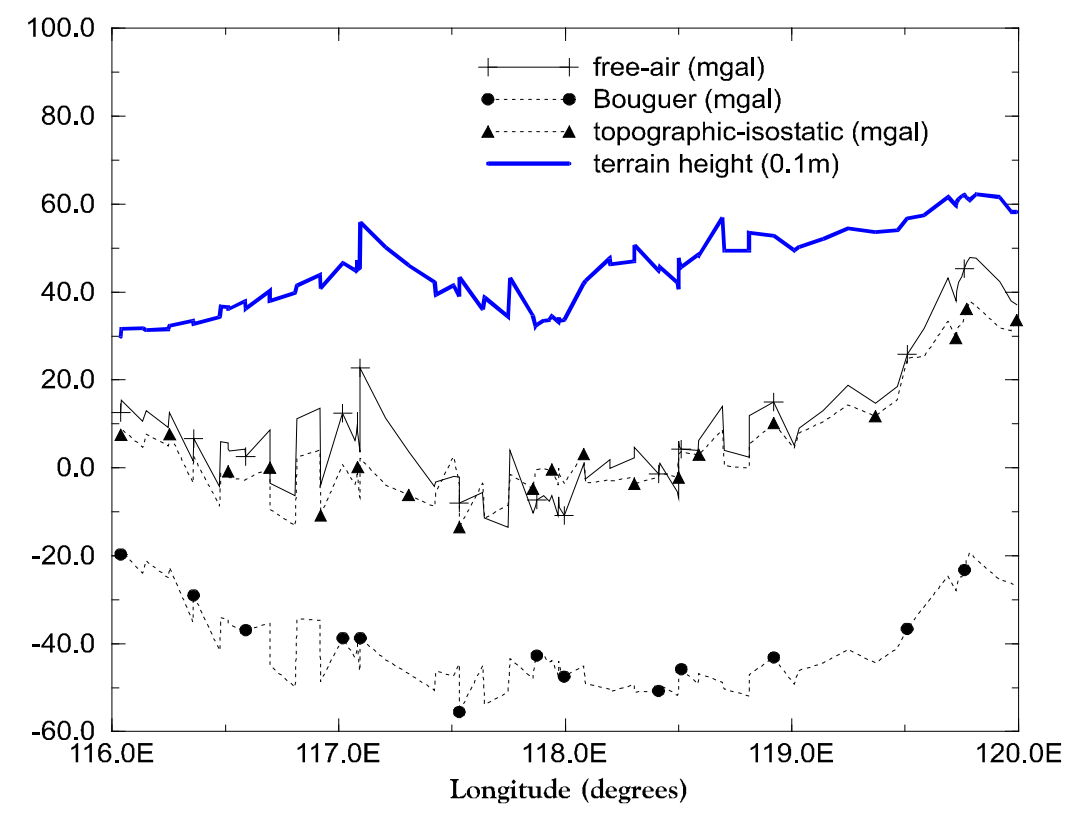

Fig. 2. Free-air, refined Bouguer and topographic-isostatic gravity anomalies and terrain along profile $P_{1}$

In Figure 2, the profiles of the free-air gravity anomalies and terrain height are very similar in shape, showing that they are highly correlated. The free-air gravity anomaly profile appears to be slightly rougher than other two types of gravity anomaly profile in this area, whereas the Bouguer gravity anomaly profile appears to be the smoothest type of gravity 
anomaly in this area. This observation agrees with the inferences made from the simple statistics (Table 3)

In Figure 3, it is revealed that the Bouguer gravity anomalies are relatively rough, with some extremely steep gradients exist in the interval $126.0^{\circ} \mathrm{E}$ to $128.0^{\circ} \mathrm{E}$ (which is corroborated by [1]). The free-air and topographic-isostatic gravity anomalies for profile $\mathrm{P}_{2}$ are slightly less correlated with terrain heights than for profile $P_{1}$ (see Figure 2). This implies that a complicated geological structure exists in this region and $2670 \mathrm{kgm}^{-3}$ is not representative of the true topographic mass-density. This was confirmed by inspection of regional geological maps, where the east-west Capricorn Orogen separates the ancient Yilgarn (south) and Pilbara (north) Cratons. The topographic-isostatic and free-air gravity anomaly profiles appear to be approximately equally smooth. These interpretations also agree with the conclusions reached from the descriptive statistics (Table 3).

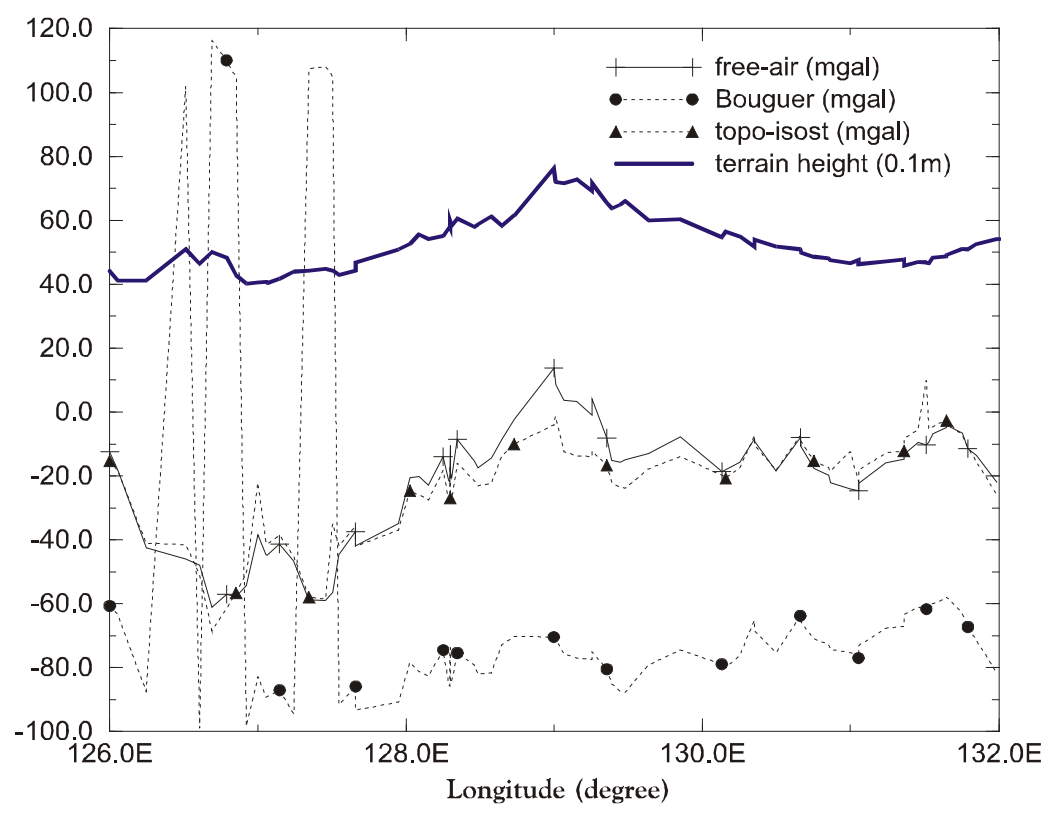

Fig. 3. Free-air, refined Bouguer and topographic-isostatic gravity anomalies and terrain along profile $\mathrm{P}_{2}$

In Figure 4, the free-air gravity anomalies are highly correlated with the terrain heights in the interval $147^{\circ} \mathrm{E}$ to $149^{\circ} \mathrm{E}$. However, all three types of gravity anomaly are not correlated with the terrain heights in the interval $145^{\circ} \mathrm{E}$ to $146.5^{\circ} \mathrm{E}$. This is most probably due to a combination of the relatively smooth terrain (due to extensive weathering) and large lateral sub-surface density variations (due to complicated geological structure) in this area. Therefore, if standard gravity reductions are applied in this region using only the terrain heights and a constant topographic density, the resulting gravity anomaly will not necessarily become smoother. Over the whole profile, the free-air gravity anomalies appear to be roughest, whereas the Bouguer gravity anomalies are smoother and the topographic-isostatic gravity anomalies are the smoothest. Again, this agrees with the conclusions reached from Table 3.

An interesting observation to be made from Figures 3 and 4 is that the terrain heights, in contrast to the gravity anomalies, are relatively smooth in some places, showing that the topography is of longer wavelength in nature than the gravity anomaly. 


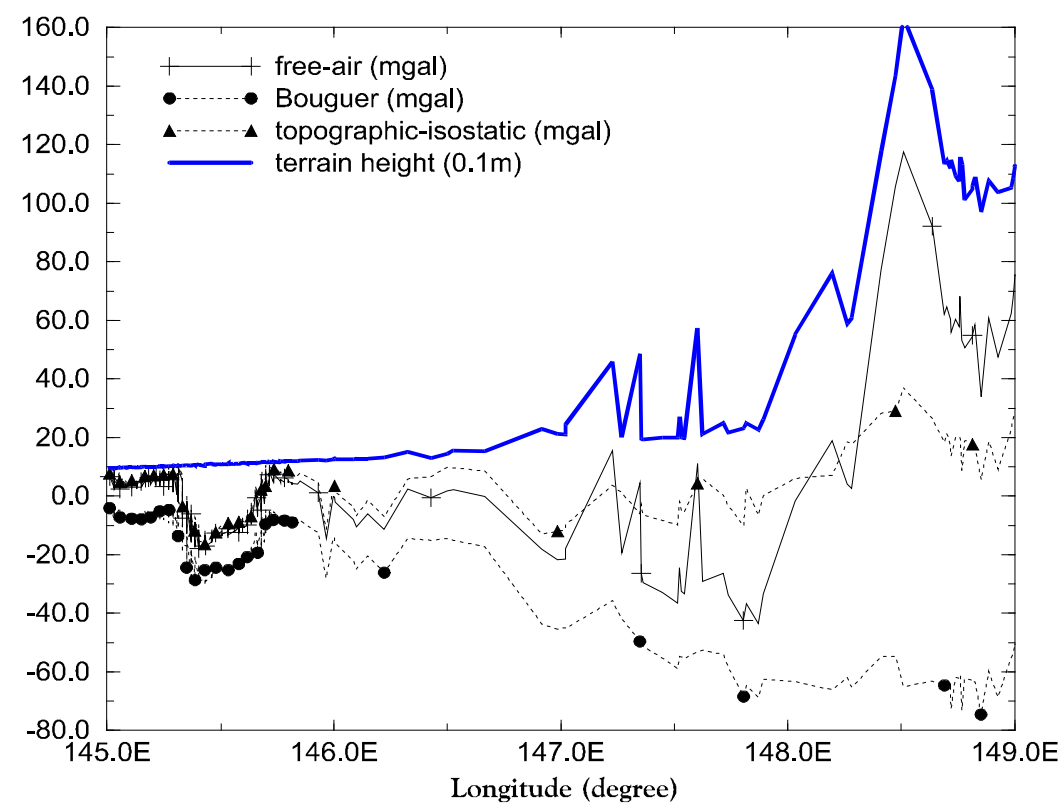

Fig. 4. Free-air, refined Bouguer and topographic-isostatic gravity anomalies and terrain along profile $P_{3}$

Therefore, it is concluded that the gravity anomalies do not always have a strong correlation with the terrain heights along these profiles, suggesting that large mass-density variations exist below these regions. From this point of view, topographical mass-density information is more important than terrain height information for smoothing the gravity anomalies in these, and probably other, areas of Australia. This is necessary because the actual vertical gradient of gravity in Australia cannot be accurately represented by the normal or Bouguer gravity gradients, thus causing high-frequency errors in the computed gravity anomalies.

Visual inspection and comparison of the profiles confirms that none of the gravity anomaly types are consistently smoothest in these regions of Australia. The conclusions made from the graphs regarding the relative smoothness of the gravity anomaly types agree with the conclusions made from the simple statistical comparisons. However, the statistical comparisons are considered superior because they are easy and efficient to implement, especially for large datasets.

\section{Power Spectral Analysis}

To analyse the spectral characteristics of the three gravity anomaly profiles, Fourier power spectral analysis [6,4] was implemented. This method can be useful for analysing the detailed spectral structure of the gravity field in terms of power distribution versus wavelength. Recall that the gridding process relies more upon the detailed features of the quantity to be interpolated. Therefore, the relative power of the gravity anomaly types in the short wavelengths should only be used to deduce the relative roughness of each, with more power indicating a greater roughness and vice versa. The power spectra of free-air, Bouguer and isostatic-topographic gravity anomalies for profiles $P_{1}, P_{2}$ and $P_{3}$ were computed using the public-domain xmgr software and are shown in Figures 5 to 7, respectively. In Figure 5, all three gravity anomaly profiles appear to contain very similar power spectra in the short wavelength components ( $\sim 5 \mathrm{~km}$ to $\sim 50 \mathrm{~km})$. 


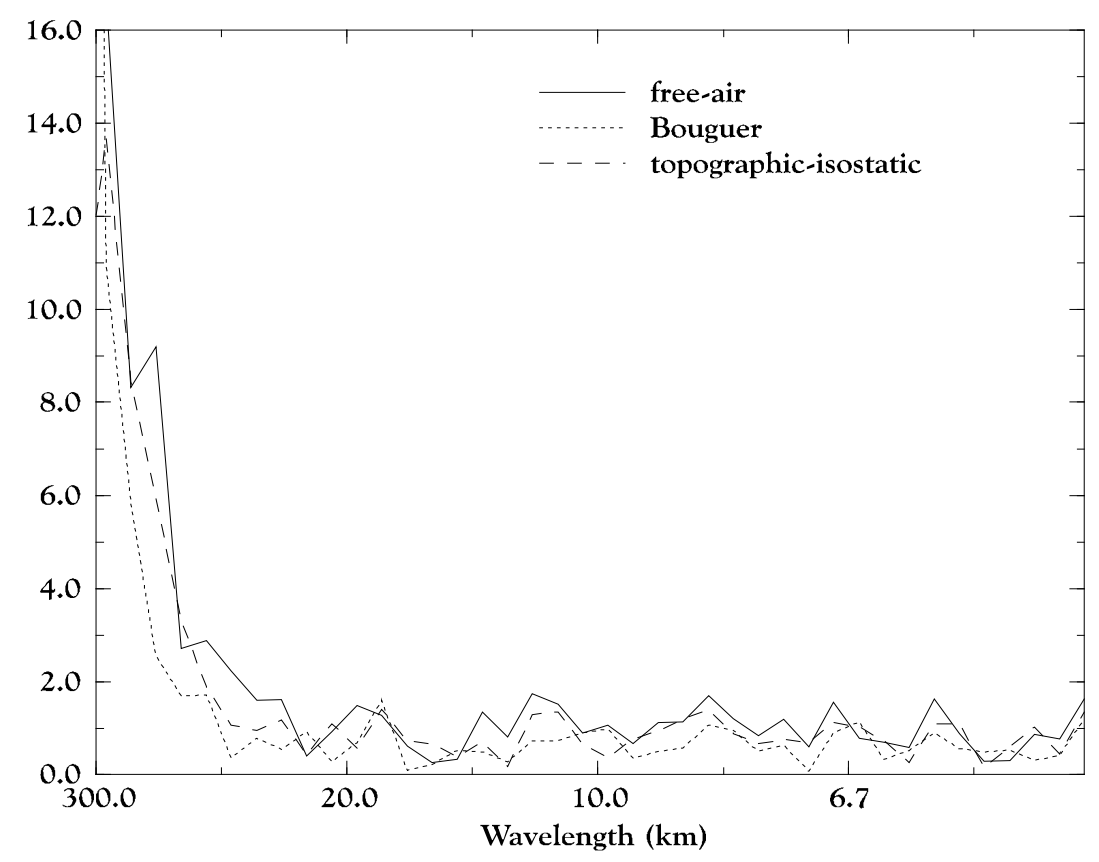

Fig. 5. Power spectra of free-air, refined Bouguer and topographic-isostatic gravity anomalies along profile $\mathrm{P}_{1}$

As such, it is difficult to determine which is the smoothest gravity anomaly type in this area using the power spectral analysis. Therefore, the statistical and visual techniques are considered to be more useful for the determination of the relative roughness of the gravity anomaly types in this region.

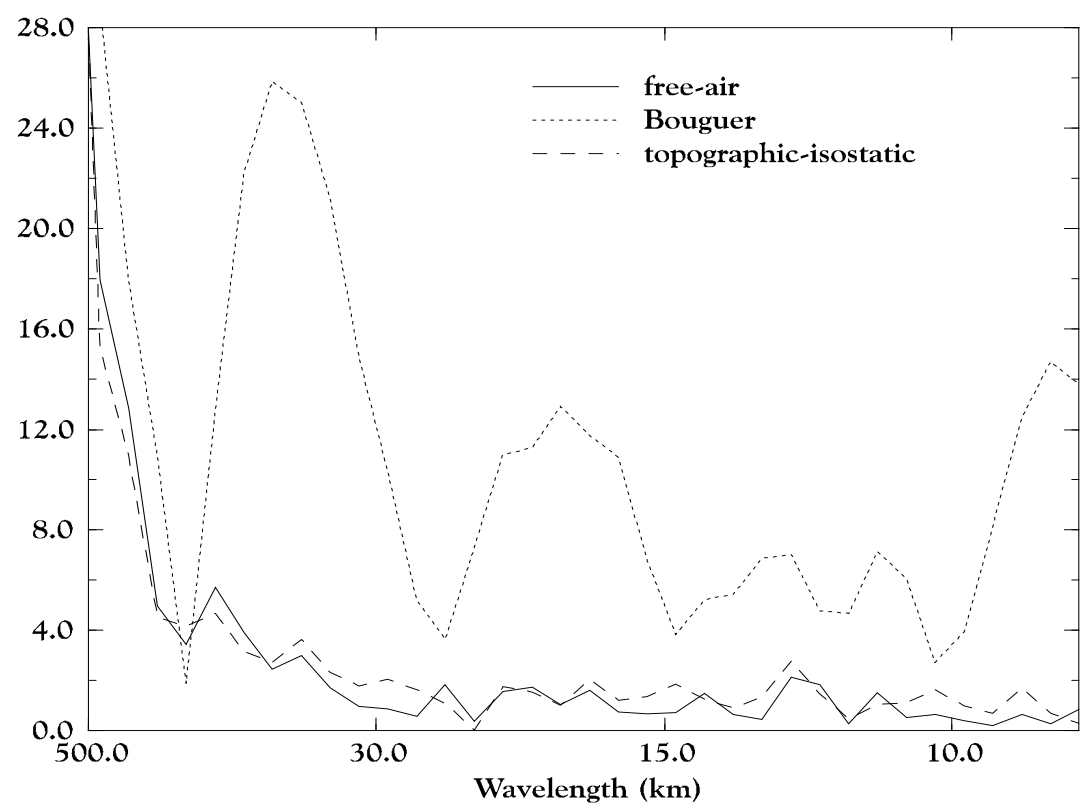

Fig. 6. Power spectra of free-air, refined Bouguer and topographic-isostatic gravity anomalies along profile $\mathrm{P}_{2}$

In Figure 6, it is clearly seen that the Bouguer gravity anomalies are much rougher than the free-air and topographic-isostatic gravity anomalies in the short and medium frequency components ( $\sim 10 \mathrm{~km}$ to $\sim 300 \mathrm{~km})$. The roughness of the free-air and topographic-isostatic gravity anomalies appears to be similar. Therefore, it is difficult to determine which is the smoother directly from Figure 6. These conclusions comply with those reached from the previous statistical and graphical comparisons. In this case, the power spectral method is only useful if one type of gravity anomaly is considerably rougher than another type. This is already clearly evident from the simple statistics (cf. Table 3), so the power spectral method 
is considered to be less effective in determining the relative roughness of all the gravity anomaly types.

From Figure 7, it is concluded that the free-air gravity anomalies are rougher than the Bouguer and topographic-isostatic gravity anomalies in the short wavelength range (less than $\sim 50 \mathrm{~km}$ ), which agrees with the conclusion reached from the simple statistical and graphical comparisons. The Bouguer and topographic-isostatic anomalies appear to be similarly smooth in this area, which does not agree with the conclusion reached from the simple statistical and graphical comparisons.

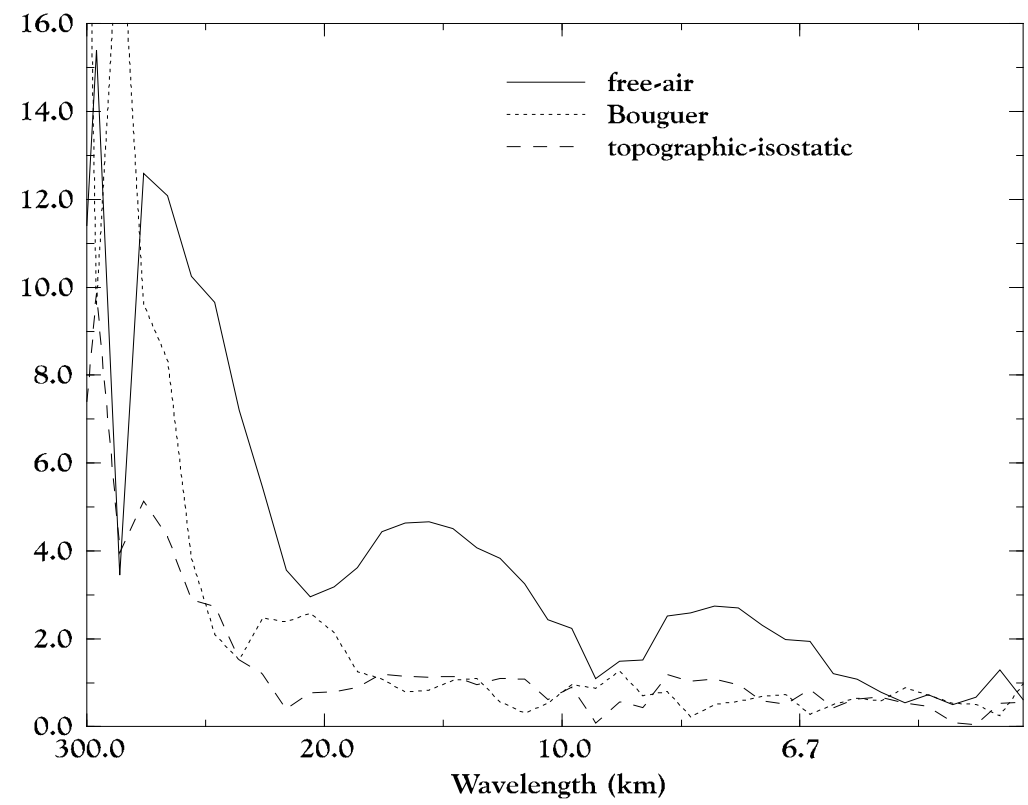

Fig. 7. Power spectra of free-air, refined Bouguer and topographic-isostatic gravity anomalies along profile $\mathrm{P}_{3}$

Power spectral analysis also shows that not one of the gravity anomaly types is consistently the smoothest at the shorter wavelengths in the test areas. However, it is less informative and conclusive than the statistical and graphical methods, because it can only reveal large differences in relative roughness, which are already obvious from the other techniques. Moreover, it also relies on graphical techniques, which make it more cumbersome to use for large, continent-wide datasets. Therefore, power spectral analysis is considered a less useful measure of the relative roughness of various types of gravity anomaly prior to gridding, though it can give more information on the detailed (short wavelength) relative roughness of the gravity anomalies.

\section{Hurst Fractal Dimension}

As stated earlier, the fractal dimension of a profile can be used to quantify its relative roughness. The Hurst fractal dimension [13] has been applied to the three gravity profiles and the results summarised in Table 4. Recall that the higher the fractal dimension, the rougher the gravity anomaly and vice versa.

Table 4. The Hurst fractal dimension of the gravity anomalies $(F A=$ free-air, $B G=$ refined Bouguer, $T I=$ topographic-isostatic) over the three test profiles

\begin{tabular}{c|c|c|c}
\hline & $\mathrm{P}_{1}$ & $\mathrm{P}_{2}$ & $\mathrm{P}_{3}$ \\
\hline FA & $1.9907 \pm 0.0026$ & $1.9985 \pm 0.0001$ & $1.9517 \pm 0.0071$ \\
\hline BG & $1.9920 \pm 0.0020$ & $1.9999 \pm 0.0000$ & $1.9616 \pm 0.0047$ \\
\hline TI & $1.9923 \pm 0.0022$ & $1.9985 \pm 0.0001$ & $1.9517 \pm 0.0071$ \\
\hline
\end{tabular}


From Table 4, the Hurst fractal dimension also indicates that not one of the types of gravity anomaly is consistently the smoothest over the three profiles. This agrees with the conclusions reached using the statistical, graphical and power spectral techniques. However, there is some disagreement in the identification of which gravity anomaly type is the smoothest or roughest along each profile. For instance, for profile $\mathrm{P}_{1}$ the Hurst fractal dimension indicates that the free-air gravity anomalies are the smoothest, whereas these are identified as the roughest from the statistical and graphical comparisons. However, when taking into consideration the regression error, the conclusion reached from the Hurst fractal analysis is not always statistically significant. Therefore, while the fractal dimension supports the notion that not one type of gravity anomaly is the smoothest, it is less effective at identifying the exact one.

\section{CONCLUSIONS}

The gravity field of Australia appears to be unusual in that it does not follow the traditional axioms of relative roughness of the various gravity anomaly types, behaving quite differently to that reported for other countries. None of the gravity anomalies appear to be consistently the smoothest over all areas of Australia. Contrary to usual expectations, the refined Bouguer and topographic-isostatic gravity anomalies are not necessary smoother than free-air gravity anomalies. This finding may also be pertinent to other parts of the world. Therefore, the relative roughness of different types of gravity anomaly should be assessed before gridding.

In some regions of Australia, observed gravity is not strongly correlated with height, but is more strongly correlated with subsurface density variations, which are associated with a complicated geological structure. This indicates that the use of the (sometimes longer wavelength) topographic information in Australia cannot always smooth the gravity field. Moreover, the omission of accurate topographic density information in the refined Bouguer reduction does not necessarily provide further smoothing of the gravity field. This peculiar feature is probably due to Australia being one of the world's oldest continents, which has a complicated geological history and a relatively smooth topography in many parts due to extensive weathering. Therefore, topographic corrections to Australian gravity data that do not use topographic density information are not necessarily useful for smoothing the gravity field prior to gridding.

The techniques used to determine the relative roughness of the gravity anomaly types comprise simple statistics, graphical visualisation, power spectral analysis and the Hurst fractal dimension. Of these four methods, the simple statistical comparison and graphical techniques appear to be the most informative and agree with one another, which is to be expected. The statistical analysis method is simple, intuitive and easily implemented, especially for large datasets. However, the usefulness of this method is dependent on the area over which the statistics are computed. For instance, the conclusion reached for the whole of Australia (Table 2) is at odds with the conclusion reached for each area (Table 3). Therefore, the optimal size of area over which the simple statistics are computed has to be determined.

The power spectral and Hurst fractal methods do not perform as well as the statistical and graphical techniques, because they can only reveal the roughest or smoothest gravity anomaly type if there is a large difference. However, power spectral analysis can give more detailed information for specific frequencies in the gravity field. The Hurst fractal method can only approximately indicate the relative roughness of the gravity anomalies because of regression errors. Moreover, the conclusions deduced from the Hurst method do not always agree with the other methods. When the smoothness of the gravity anomaly types is similar, the power spectral and Hurst fractal methods do not perform very well. Therefore, the simple statistical (and graphical) comparisons are recommended for the analysis of the relative roughness of gravity anomalies prior to gridding gravity data.

It is also recommended that the smoothest type of gravity anomaly be identified using statistical or graphical comparisons on a region-by-region basis, and then used for gravity gridding prior to subsequent geoid computations. 


\section{ACKNOWLEDGMENTS}

We wish to thank the Australian Geological Survey Organisation and Australian Surveying and Land Information Group (now Geoscience Australia) for kindly providing the gravity and terrain data. This research is partly supported by the Victorian Partnership for Advanced Computing (VPAC) Expertise Program Grant Scheme (grant no: EPPNRM035/2001) and the Department of Geospatial Science, RMIT University (Zhang). Thanks are also extended to the Australian Research Council for earlier funding of this project through large grant A39938040 (Featherstone).

\section{References}

1. Anfiloff, V. (1982) Elevation and gravity profiles across Australia: some implications for tectonism, BMR Journal of Australian Geology and Geophysics, 7, 47-54.

2. Barnsley, M. (1988) Fractals Everywhere, Boston Academic Press, Boston, 394 pages.

3. Barton, C. and Pointe, R.P.L. (1995) Fractals in the Earth Sciences, Plenum Press, New York, 265 pages.

4. Bracewell, R.N. (1986a) The Fourier Transform and its Applications (second edition), McGrawHill, New York, 474 pages.

5. Bracewell, R.N. (1986b) The Hartley Transform, Oxford University Press, Oxford, 160 pages.

6. Brigham, E.O. (1988) The Fast Fourier Transform, Prentice-Hall, London, 252 pages.

7. Farge, M., Hunt, J.C.R. and Vassilicos, J.C. (eds.) (1993) Wavelets, Fractals and Fourier Transforms, Clarendon Press, Oxford, 403 pages.

8. Featherstone, W.E., Kearsley, A.H.W. and Gilliland, J.R. (1997) Data preparations for a new Australian gravimetric geoid, The Australian Surveyor, 42(1), 33-44.

9. Forsberg, R. (1984) A study of terrain reduction, density anomalies and geophysical inversion methods in gravity field approximation, Report No. 355, Department of Geodetic Science and Surveying, Ohio State University, Columbus, 129 pages.

10. Hurst, H. E., Black, R.P. and Simaika, Y.M. (1965) Long Term Storage: An Experimental Study, Constable, London, 145 pages.

11. Li, Y.C., Sideris, M.G. and Schwarz, K-P. (1995) A numerical investigation on height anomaly prediction in mountainous areas, Bulletin Géodésique, 69, 143-156.

12. Mandelbrot, B. and Benoit, B. (1982) The Fractal Geometry of Nature, W. H. Freeman \& Co., San Francisco, 468 pages.

13. Russ, J.C. (1994) Fractal Surfaces, Plenum Press, New York, 309 pages.

14. Schwarz, K.P., Sideris, M.G. and Forsberg, R. (1990) The use of FFT techniques in physical geodesy, Geophysical Journal International, 100, 485-514.

15. Smith, W.H.F. and Wessel, P. (1990) Gridding with Continuous Curvature Splines in Tension, Geophysics, 55, 293-305.

16. Tziavos, I.N. (1996) Comparisons of spectral techniques for geoid computations over large regions, Journal of Geodesy, 70, 357-373.

17. Zhang, KF (1997) An evaluation of FFT geoid determination techniques and their application to Australian GPS heighting, Ph.D. Thesis, School of Surveying and Land Information, Curtin University of Technology, Australia, 261 pages. 